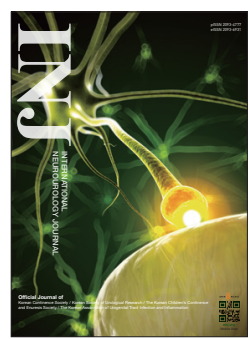

\title{
Differential Effects of Alpha 1-Adrenoceptor Antagonists on the Postsynaptic Sensitivity: Using Slice Patch-Clamp Technique for Inhibitory Postsynaptic Current in Substantia Gelatinosa Neurons From Lumbosacral Spinal Cord in Rats
}

\author{
Daisuke Uta ${ }^{1,2 \star}$, Tsuyoshi Hattori ${ }^{3 \star}$, Megumu Yoshimura ${ }^{2,4,5}$ \\ ${ }^{1}$ Department of Applied Pharmacology, Faculty of Pharmaceutical Sciences, Toyama, Japan \\ ${ }^{2}$ Department of Integrative Physiology, Graduate School of Medical Sciences, Kyushu University, Fukuoka, Japan \\ ${ }^{3}$ Department of Medical Affairs, Asahi Kasei Pharma Co., Tokyo, Japan \\ ${ }^{4}$ Graduate School of Health Sciences, Kumamoto Health Science University, Kumamoto, Japan \\ ${ }^{5}$ Nogata Nakamura Hospital, Fukuoka, Japan
}

Purpose: Alpha1-adrenoceptors participate in improving storage symptoms of male lower urinary tract symptoms. However, the mechanism of action of these compounds remains unclear. The goal of the present study was to clarify the effect of $\alpha 1-$ adrenoceptor antagonists on $\gamma$-aminobutyric acid (GABA)/glycine-mediated outward currents of the inhibitory postsynaptic current (IPSC) in substantia gelatinosa (SG) neurons from the lumbosacral spinal cord in rats.

Methods: Male adult Sprague-Dawley rats were used. Blind whole-cell patch-clamp recordings were performed in SG neurons from isolated spinal cord slice preparations. IPSCs were recorded in individual SG neurons to which naftopidil (100 $\mu \mathrm{M})$, tamsulosin $(100 \mu \mathrm{M})$, silodosin $(30 \mu \mathrm{M})$, or prazosin $(10 \mu \mathrm{M})$ were applied sequentially with intervening washout periods. Strychnine $(2 \mu \mathrm{M})$, bicuculline $(10 \mu \mathrm{M})$, or tetrodotoxin $(\mathrm{TTX})(1 \mu \mathrm{M})$ were added before naftopidil. Individual outward currents were analyzed.

Results: The bath application of naftopidil, yielded outward IPSCs in 13 of 52 SG neurons. The naftopidil response was unchanged in the presence of TTX. Regression analysis of the outward currents between the 1st and 2nd applications of naftopidil revealed a Pearson correlation coefficient of 0.996 with a line slope of 0.983 . The naftopidil-induced outward current was attenuated in the presence of strychnine and/or bicuculline. The GABA/glycine-mediated outward currents induced by tamsulosin, silodosin, and prazosin were smaller than those obtained with naftopidil.

Conclusions: Naftopidil-induced GABA/glycine-mediated outward currents in a subset of SG neurons prepared from the L6S1 level of rat spinal cord. The results indicated that $\alpha 1$-adrenoceptor antagonists, particularly naftopidil, induce neural suppression (in part) by mediating hyperpolarization. The response is associated with glycinergic and/or GABAergic neural transmission. Naftopidil may suppress the micturition reflex and improve urinary storage symptoms as a subsidiary effect resulting from hyperpolarization in SG neurons of the spinal cord.

Keywords: Adrenergic alpha-1 receptor antagonists; Inhibitory postsynaptic potentials; Naftopidil; Substantia gelatinosa

Corresponding author: Daisuke Uta (iD https://orcid.org/0000-0001-5644-1348 Department of Applied Pharmacology, Faculty of Pharmaceutical Sciences, University of Toyama, Toyama 930-0194, Japan

E-mail: daicarp@pha.u-toyama.ac.jp

Co-corresponding author: Tsuyoshi Hattori iDhttps://orcid.org/0000-0001-6425-3945

Department of Medical Affairs, Asahi Kasei Pharma Co., Tokyo 100-0006, Japan

E-mail: hattori.tg@om.asahi-kasei.co.jp

${ }^{*}$ Daisuke Uta and Tsuyoshi Hattori contributed equally to this study as co-first authors.

Submitted: December 18, 2019 / Accepted after revision: March 30, 2020
This is an Open Access article distributed under the terms of the Cretive Commons Attribution Non-Commercial License (https://creativecommons.org/licenses/by-nc/4.0/) which permits unrestricted non-commercial use, distri-
bution, and reproduction in any medium, provided the original work is properly cited. 
- Grant/Fund Support: This work was supported by grants from the programs for Grants-in-Aid for Scientific Research (DU and MY) of the Ministry of Education, Science, Sports and Culture of Japan (Grant Numbers JP19K09323, JP15K08667, JP25860431, and JP21600005), and was supported in part by the MEXT-Supported Program for the Strategic Research Foundation at Private Universities, "Creation of 3D nanomicro structures and its application to biomimetics and medicine", during 2015-2019 (DU, Grant Number S1511031). This study also was supported in part by the Asahi Kasei Pharma Corporation.

- Research Ethics: All experiments were performed in accordance with the "Guiding Principles for Care and Use of Animals in the Field of Physiological Sciences" of the Physiological Society of Japan and were approved by the local Animal Experiment Committee of the Kumamoto Health Science University and Kyushu University. All efforts were made to minimize animal suffering and the number of animals used for the studies.

- Conflict of Interest: TH is an employee of Asahi Kasei Pharma Corporation. Others have no potential conflict of interests relevant to this article.

\section{- HIGHLIGHTS \\ - Naftopidil reproducibly induces outward current of IPSC in a subset of substantia gelatinosa neurons. \\ - The naftopidil response is mediated by glycinergic and/or GABAergic neural transmission. \\ - Other a1-adrenoceptor antagonists, particularly prazosin, yield weaker changes in the outward current.}

\section{INTRODUCTION}

In elderly men, lower urinary tract symptoms (LUTS) are related to benign prostatic obstruction/benign prostatic hyperplasia (BPH), bladder dysfunction including overactive bladder, and nocturnal polyuria [1]. Although LUTS/BPH typically is not a life-threatening condition, the impact of LUTS/BPH on the quality of life can be significant and should not be underestimated [2]. To manage male LUTS pharmacologically, a1adrenoceptor blockers are well-established drugs and are prescribed widely. a1-Adrenoceptor antagonists relieve the voiding symptoms of LUTS by relaxing smooth muscle in the urethra and prostate, resulting in reduction of enhanced tonus or contractility, thus counteracting the lowered urine flow rates [3]. Alpha1-adrenoceptors also participate in improving the storage symptoms of LUTS [4], although the mechanism of action of the compounds in this context remains unclear. In rats subjected to conscious cystometry, tamsulosin, naftopidil, and silodosin all have been shown to prolong the micturition interval [5]. Clinical studies comparing the effects of a1-adrenoceptor antagonists, naftopidil, and tamsulosin yielded contradictory results in equivalence for the International Prostate Symptom Score [6], but yielded consistent differences for the volume at first desire to void and at maximum desire to void in the urodynamic study [7].

The micturition reflex is believed to be mediated by neuronal circuits in the lumbosacral spinal cord [8-10]. It has been suggested that a1-adrenoceptor antagonists suppress the micturition reflex via action in the spinal cord by isovolumetric cystometry [11], employing a mode of action of naftopidil that requires functional upregulation of $\gamma$-aminobutyric acid (GABA) and/or glycine receptors at the lumbosacral level [12]. By using voltage-clamp recordings, it is possible to identify primary afferent information in substantia gelatinosa (SG, lamina II of Rexed) neurons [13] mediated by different primary afferent fibers $[14,15]$. The efficacy of synaptic transmission is determined by presynaptic neurotransmitter release probability and postsynaptic responsiveness, which are evaluated as the frequency and amplitude of miniature excitatory or inhibitory postsynaptic current (mEPSC or mIPSC), respectively. Patchclamp recording of spinal slices has been used to measure mEPSC and mIPSC, revealing that presynaptic firing corresponds to postsynaptic depolarization and hyperpolarization, respectively. Analyses of mIPSCs have provided extensive information of experimental interest, as follows. Increments in the frequencies of mIPSCs mean that stimuli act on presynaptic sites, leading to the release of inhibitory neurotransmitters. Increments in the amplitudes of mIPSCs mean that stimuli act on postsynaptic sites, leading to upregulation of the sensitivity of the action sites to inhibitory neurotransmitters. Changes of membrane current indicate outward or inward orientation of the current (hyperpolarization or depolarization) regarding inhibitory neuronal transmissions [16]. Generally, outward IPSCs indicate an influx of $\mathrm{Cl}^{-}$, which induces hyperpolarization.

A previous study facilitated our understanding of the effect of naftopidil on the micturition reflex [17], indicating that naftopidil increases the frequency of mIPSCs by activating inhibitory interneuron terminals in the spinal dorsal horn [17]. However, information on outward/inward currents in IPSCs and differences induced by various $\alpha 1$-adrenoceptor antagonists (e.g., naftopidil, tamsulosin, and silodosin) remain unclear, naftopidil was the first $\alpha 1$-adrenoceptor antagonist evaluated in in- 
vestigation of the mechanism of micturition reflex in the lumbosacral cord. Our primary goal was to determine the effects of an $\alpha 1$-adrenoceptor antagonist, naftopidil, on outward/inward currents in IPSCs. The secondary goals were to determine the differences between the outward currents induced by naftopidil and the other a1-adrenoceptor antagonists, and to determine whether the outward current induced by naftopidil mediates GABA/glycine receptor activation in vitro in SG neurons derived from rat lumbosacral spinal cord.

\section{MATERIALS AND METHODS}

\section{Spinal Cord Slice Preparation}

The methods for obtaining slices of the adult rat spinal cord and for blind patch-clamp recordings from SG neurons have been described in detail elsewhere [17-20]. Briefly, adult male SpragueDawley rats (6-8 weeks old) were deeply anesthetized with urethane $(1.2 \mathrm{~g} / \mathrm{kg}$, intraperitoneally), and a lumbosacral laminectomy then was performed. The lumbosacral segments of the spinal cord (L2-S3) with ventral and dorsal roots were removed and placed in ice-cold Krebs solution equilibrated with 95\% $\mathrm{O}_{2}-5 \% \mathrm{CO}_{2}$. The Krebs solution contained (in $\mathrm{mM}$ ): $\mathrm{NaCl} 117$, $\mathrm{KCl}$ 3.6, $\mathrm{CaCl}_{2} 2.5, \mathrm{MgCl}_{2} 1.2, \mathrm{NaH}_{2} \mathrm{PO}_{4} 1.2, \mathrm{NaHCO}_{3} 25$, and glucose $11(\mathrm{pH}, 7.4)$. Immediately after removal of the spinal cord, the rats were killed by exsanguination under urethane anesthesia. The pia-arachnoid membrane was removed after cutting all the ventral and dorsal roots. The spinal cord was mounted on a vibratome, and a $500-\mu \mathrm{m}$-thick transverse slice with the attached dorsal root was cut. The slice was placed on a nylon mesh in the recording chamber in a volume of $0.5-\mathrm{mL}$ Krebs solution, and was completely submerged and perfused with Krebs solution saturated with $95 \% \mathrm{O}_{2}-5 \% \mathrm{CO}_{2}$ at $37 \pm 1^{\circ} \mathrm{C}$ and a flow rate of $10-15 \mathrm{~mL} / \mathrm{min}$.

\section{Whole-Cell Patch-Clamp Recordings From SG Neurons}

The SG was easily discernible with transmitted illumination as a relatively translucent band across the dorsal horn in the transverse slice preparations. Blind whole-cell voltage-clamp recordings were made from SG neurons, as described previously $[17,18,20,21]$. The patch pipettes were filled with a solution containing cesium solution (in mM): $\mathrm{Cs}_{2} \mathrm{SO}_{4} 110$, tetraethylammonium (TEA) $5, \mathrm{CaCl}_{2} 0.5, \mathrm{MgCl}_{2}$, ethylene glycol tetraacetic acid 5, 4-(2-hydroxyethyl)-1-piperazineethanesulfonic acid 5, and adenosine 5'-triphosphate- $\mathrm{Mg} 5$ (pH, 7.2). With the cesium pipette solution, a holding potential was easily depolarized to $0 \mathrm{mV}$, since the $\mathrm{K}^{+}$channels were blocked by cesium and TEA. The tip resistance of the patch pipettes was 6-12 M 2 . Series resistance was assessed according to the response to a 5-mV hyperpolarizing step. This value was monitored during the recording session, and data were rejected if values changed by $>15 \%$. Signals were acquired with a patch-clamp amplifier (Axopatch 700A, Molecular Devices, Union City, CA, USA). The data were digitized with an AD/DA converter (Digidata 1321A, Molecular Devices), stored on a personal computer using a data acquisition program (Clampex, version 9.0, Molecular Devices), and analyzed using a software package (Clampfit, version 9.0, Molecular Devices). Cell recordings were made in voltage-clamp mode at holding potentials of $0 \mathrm{mV}$ to record IPSCs $[17,18,20]$. We used a total of 17 animals in this study. One slice was collected from each rat and recordings were obtained for 1-5 cells/slice. One to 5 cells were recorded for each set of experiments.

\section{Drug Application}

Naftopidil (final concentration, 100 $\mu$ M) (PubChem CID: 4418) (Asahi Kasei Pharma Co., Tokyo, Japan) was dissolved in 1\% dimethyl sulfoxide (PubChem CID: 679) (Wako, Osaka, Japan) in Krebs solution. Tamsulosin $(100 \mu \mathrm{M})$ and silodosin $(30 \mu \mathrm{M})$ were dissolved in Krebs solution. The other compounds used in

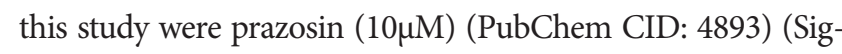
ma-Aldrich, St. Louis, Mom USA), tetrodotoxin (TTX) $(1 \mu \mathrm{M})$ (PubChem CID: 6324668) (Wako), strychnine $(2 \mu \mathrm{M})$ (PubChem CID: 441071) (Sigma-Aldrich), and bicuculline (10 $\mu \mathrm{M})$ (PubChem CID: 10237) (Sigma-Aldrich). All drugs were applied by sequential perfusion in individual cells with washout periods via a three-way stopcock without changes in the perfusion rate or temperature. The application schedule was described previously [22].

\section{Statistical Analysis}

Statistical analysis was performed using JMP ver. 14 (SAS Institute, Cary, NC, USA). Continuous values are revealed mean \pm standard error of the mean. Inferential statistical analysis was not performed, because the number of SG neurons that exhibited induced outward current was too small. Regarding reproducibility for the outward current induced by naftopidil, correlations were subjected to linear regression analysis, and the values of $r^{2}$ and the slopes of regression lines were calculated between the 1st and 2nd bath applications in a given neuron. Pearson correlation coefficients (r-values) were analyzed. 


\section{RESULTS}

\section{Outward/Inward Current of IPSC in SG Neurons Following Bath Application of Naftopidil}

Bath application of naftopidil-induced outward current of IPSC in 13 of 52 SG neurons; typical charts are shown in Fig. 1. The observation of the current was reproducible (Fig. 2). Regression analysis of the currents yielded a Pearson correlation coefficient of 0.996 when comparing between the 1st and 2nd applications of naftopidil; the data plotted as a line with a slope of 0.983 . The naftopidil-induced outward current also was observed in the presence of TTX (Fig. 3). Inward current of IPSC was not observed in any SG neurons following naftopidil exposure.

\section{Impact of Glycinergic and GABAergic System for GABA/ Glycine-Mediated Outward Current of IPSC Generated by Naftopidil in SG Neurons}

The outward current induced by naftopidil in each SG neuron was attenuated in the presence of strychnine (from $28.4 \mathrm{pA} \pm 5.0$

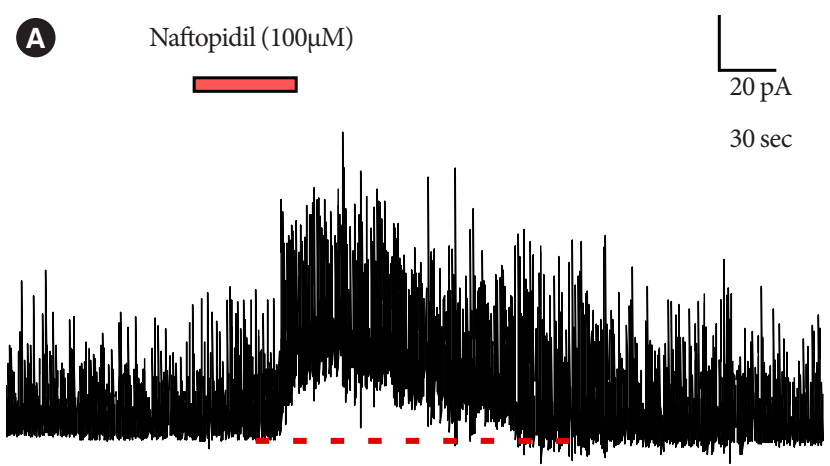

B Naftopidil $(100 \mu \mathrm{M})$

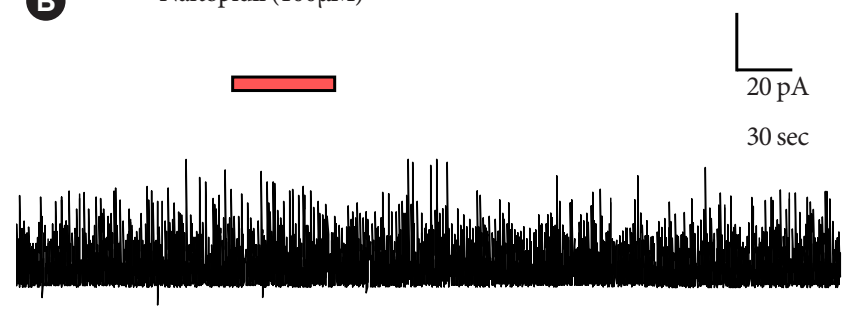

Fig. 1. Typical traces of IPSCs are provided from blind whole-cell patch-clamp recording using 2 separate $S G$ neurons from an adult rat spinal dorsal horn. (A) With outward current and mild facilitation of frequency of IPSC following bath application of naftopidil $(100 \mu \mathrm{M})$ in a SG neuron. (B) Without outward current. Bars (red) show duration of naftopidil application. IPSC, inhibitory postsynaptic current; SG, substantia gelatinosa. Horizontal dotted line indicates the holding current level in the control.
$\mathrm{pA}$ to $15.8 \mathrm{pA} \pm 2.2 \mathrm{pA}, \mathrm{n}=5$ ) or bicuculline (from $28.4 \mathrm{pA} \pm 5.0$ $\mathrm{pA}$ to $9.8 \mathrm{pA} \pm 1.6 \mathrm{pA}, \mathrm{n}=5$ ) (Fig. 4). When both strychnine and bicuculline were applied simultaneously, no naftopidil-induced outward current was detected ( $\mathrm{n}=5$ ) (Fig. 4).

\section{Effects of Other a1-Adrenoceptor Antagonists on the GABA/Glycine-Mediated Outward Current of IPSC}

Next, the effects of other a1-adrenoceptor antagonists were tested (separately) in 6 SG neurons that exhibited naftopidil-induced GABA/glycine-mediated outward current. In a subset of 3 naftopidil-responsive SG neurons, only 1 of the 3 exhibited outward current in response to tamsulosin or silodosin; notably, the same neuron responded to both compounds. In the remaining 3 naftopidil-responsive SG neurons, prazosin did not induce an outward current (Fig. 5).

\section{(A)}

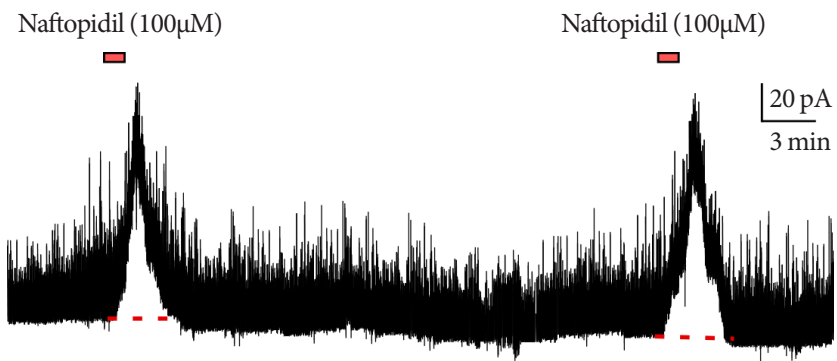

B

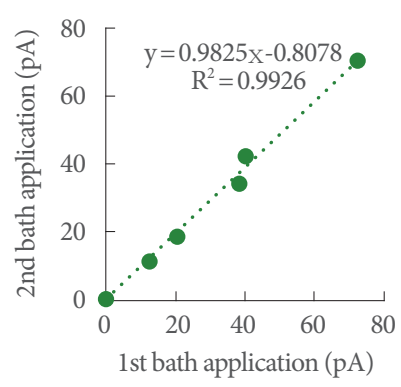

C

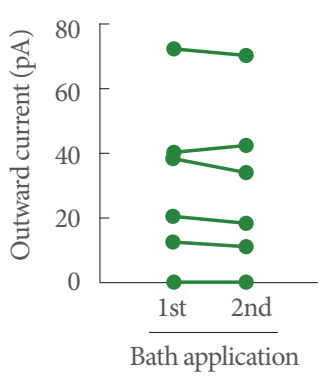

Fig. 2. Outward currents of IPSC induced by naftopidil are reproducible in SG neurons. (A) The outward currents were measured 2 times (with an intervening washout period) in the same neuron. (B) The result of a regression analysis of outward currents for the 2nd bath application compared to the 1st application in the respective neuron. Pearson correlation coefficient for the currents between the 1st and 2nd administrations of naftopidil was 0.996 , and the slope of the line was 0.983 . (C) Reproducibility of the outward current induced by naftopidil is shown for individual neurons. Bars (red) show duration of naftopidil application. IPSC, inhibitory postsynaptic current; SG, substantia gelatinosa. Horizontal dotted lines indicate the holding current level in the control. 
A

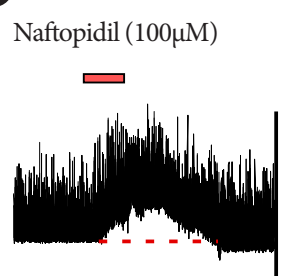

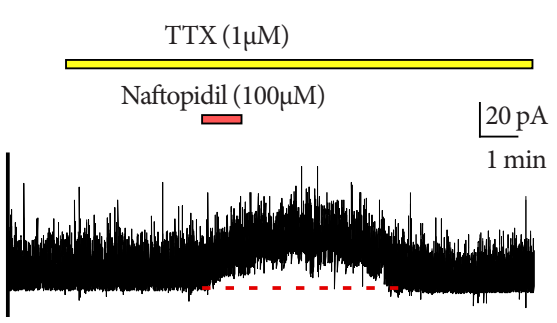

B

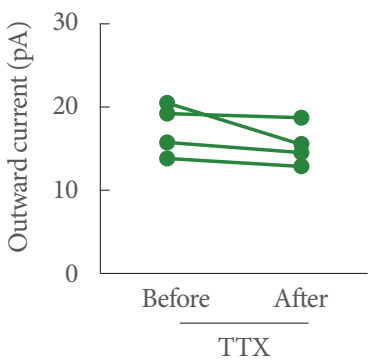

Fig. 3. Outward current of IPSC induced by naftopidil in one SG neuron was reproducible in the presence of TTX. (A) A typical trace of the outward currents is shown for one SG neuron. (B) The effect of TTX on outward currents induced by naftopidil exposure is shown in individual neurons. Bar (yellow) shows duration of TTX exposure, and bars (red) show durations of naftopidil application. IPSC, inhibitory postsynaptic current; SG, substantia gelatinosa; TTX, tetrodotoxin. Horizontal dotted lines indicate the holding current level in the control.

A

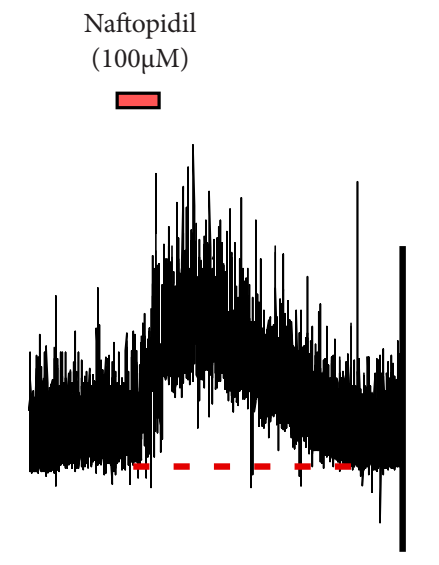

B

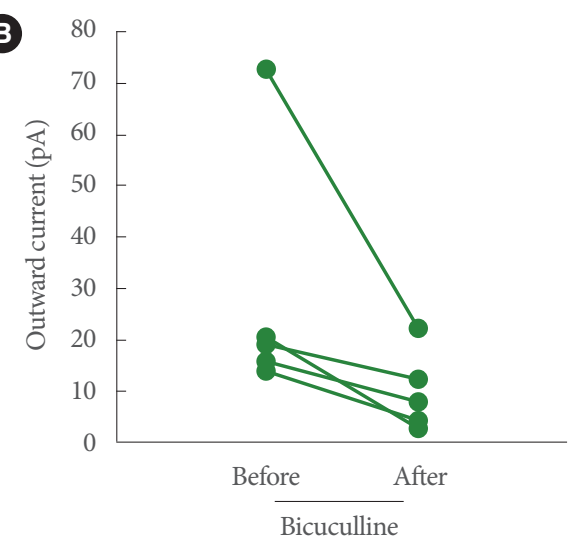

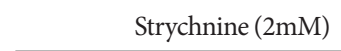

Naftopidil

$(100 \mu \mathrm{M})$

$\square$
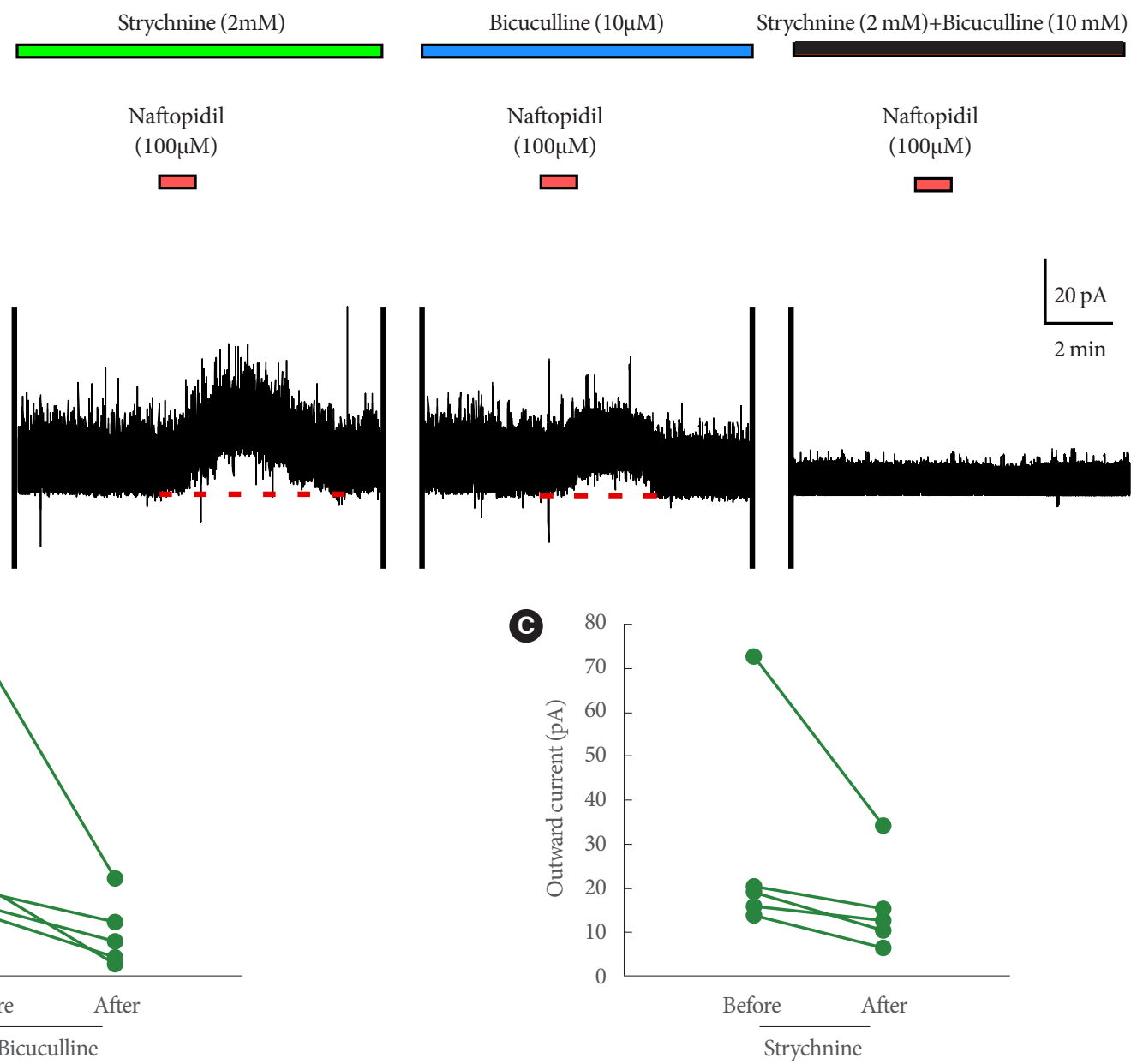

Fig. 4. The effects of strychnine and/or bicuculline on outward currents of IPSCs induced by naftopidil. Outward current induced by naftopidil in SG neurons was attenuated by prior exposure to bicuculline or strychnine (applied into the bath prior to application of naftopidil). (A) Outward current induced by naftopidil was decreased by bicuculline or strychnine in SG neurons. The effects of bicuculline (B) and strychnine (C) are shown in individual SG neurons. Bars (green, blue, and black) show durations of exposures to strychnine $(2 \mu \mathrm{M})$, bicuculline $(10 \mu \mathrm{M})$, and strychnine and bicuculline, respectively. Bars (red) shows duration of naftopidil (100 $\mu \mathrm{M})$ application. IPSC, inhibitory postsynaptic current; SG, substantia gelatinosa. Horizontal dotted lines indicate the holding current level in the control. 


\section{A}
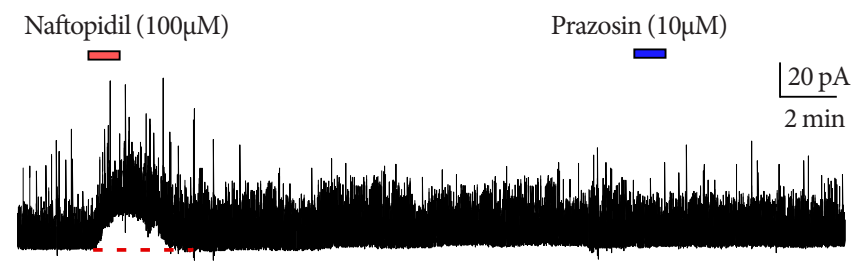

B
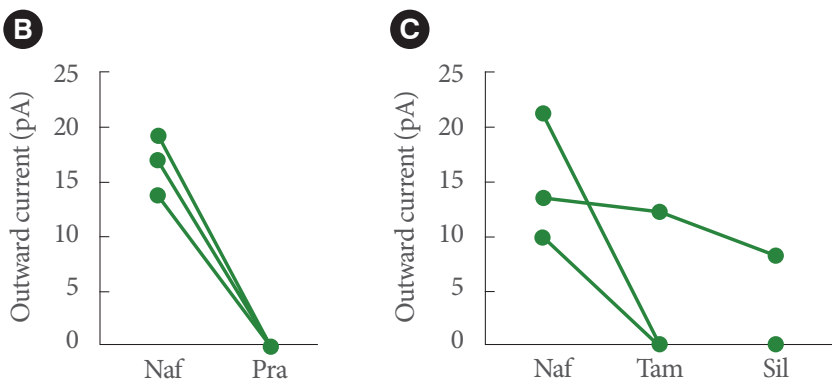

Fig. 5. Effects of a1-adrenoceptor antagonists on outward current of IPSC in SG neurons. Naftopidil (Naf) induced stronger responses than did the other tested antagonists. (A) A typical trace of the outward currents is shown. The effects (on the outward currents in individual neurons) of prazosin (Pra) (B), tamsulosin (Tam), and silodosin (Sil) (C) following naftopidil are shown. Bars (red and blue) show durations of naftopidil $(100 \mu \mathrm{M})$ and prazosin $(10 \mu \mathrm{M})$ application, respectively. IPSC, inhibitory postsynaptic current; SG, substantia gelatinosa. Horizontal dotted line indicates the holding current level in the control.

\section{DISCUSSION}

In the present study, only a subset of SG neurons was found to be naftopidil responsive. The outward current induced by naftopidil was observed reproducibly and was not antagonized by TTX (the primary endpoint). The outward current was suppressed by strychnine and/or bicuculline, and the current was evoked at lower probabilities by tamsulosin and silodosin than by naftopidil (the secondary endpoints). Prazosin did not induce an outward current in SG neurons know to be naftopidil responsive. The inward current was not observed by naftopidil.

\section{The Effects of Naftopidil on the Outward Current in IPSC and the GABA/Glycine-Mediated Effects in SG Neurons}

Regarding with the primary endpoint, naftopidil-induced outward current of IPSCs in a subset of SG neurons. It has been speculated that this variability in neuron responsivity reflects classification of SG neurons into (at least) 4 types [20], such that only certain SG neurons are naftopidil responsive. We infer that naftopidil secondarily suppresses postsynaptic neuronal activity. Since the ability of naftopidil to induce the outward current likely was not altered in the presence of TTX (Fig. 3B), the current presumably is not associated with sodium channels in the synapse.

Regarding with the secondary endpoint, the outward current induced by naftopidil was attenuated by pre-exposure to strychnine and/or bicuculline before naftopidil. These data suggest that naftopidil induces the GABA/glycine-mediated outward current (i.e., hyperpolarization) via glycinergic and/or GABAergic neurotransmission [17]. This interpretation is supported by a previous report that showed that strychnine and bicuculline eliminated the frequency of mIPSCs that was induced by naftopidil [17]. As shown in Fig. 1, mild facilitation of frequency was observed for IPSCs together with outward current in the SG neuron; this result indicated that induction of the SG neurons likely is suppressed by naftopidil, perhaps via enhancement of presynaptic release of GABA and/or glycine in addition to hyperpolarization. Therefore, naftopidil may suppress neural activity multimodally. A subpopulation of naftopidil-responsive SG neurons did not exhibit GABA/glycine-mediated outward currents of IPSCs upon exposure to tamsulosin, silodosin, or (notably) prazosin. The basis of this distinction in antagonist sensitivity is unclear, but it is possible that one or more $\alpha 1$ adrenoceptor antagonists, or conceivably the a1-adrenoceptor itself, are excluded from the site of action for induction of the GABA/glycine-mediated outward current of IPSCs. Naftopidil may attenuate bladder overactivity by enhancing fine control of excess or abnormal urge signals in the spinal cord, altering hyperpolarization in SG neurons.

\section{The Role of GABA/Glycine-Mediated Outward Current of IPSC}

IPSC is induced by inhibitory neurotransmitters (e.g., glycine and GABA) that are released from presynaptic terminals, resulting in an influx of $\mathrm{Cl}^{-}$into the postsynaptic neuronal cells. Decreasing of the membrane potential of the postsynaptic cells by $\mathrm{Cl}^{-}$influx generates an outward current. Simultaneously, hyperpolarization is observed, and the responses of postsynaptic cells are suppressed. In rat, the infusion of $0.1 \%$ acetic acid into the urinary bladder induces a nociceptive transmission, as revealed by upregulation of c-Fos expression at the L6 level of the spinal cord [23]. Acetic acid also induces shortening of the inter contraction interval in rats subjected to conscious cystometry [24]. Those investigations indicated that interruption of supra- 
spinal modulation enhances nociceptive transmission in the lower urinary tract [23]. A portion of the superficial neurons receives both nociceptive and nonnociceptive inputs from the lower urinary tract, as shown by upregulation of c-Fos expression [25]. Those results indicated the possibility that a primary afferent signal is transmitted from the bladder epithelium to the superficial SG neurons in the spinal cord. Therefore, outward currents and IPSCs in the SG neurons can be considered reasonable targets for exploring the pathology and treatment of LUTS.

\section{Extrapolation of Induction of GABA/Glycine-Mediated Outward Current of IPSC to Micturition}

GABA/glycine-mediated outward current of IPSC, which reflects hyperpolarization, is one mechanism for suppressing signal transmission by neuronal cells. The nociceptive stimuli are suppressed by hyperpolarization with resulting decreases in the excitability of SG neurons [26]. These effects could suppress the micturition reflex at the spinal cord. In the context of LUTS with pain (e.g., painful bowel syndrome), the importance of GABA/glycine-mediated outward current of IPSC will need to be further investigated. These concepts may clarify the mechanism of pathogenesis and evaluation of treatments for these symptoms.

In conclusion, a subpopulation of rat SG neurons, prepared from the L6-S1 level of the spinal cord, were shown to exhibit GABA/glycine-mediated outward current of IPSC in response to naftopidil exposure. This result suggested that $\alpha 1$-adrenoceptor antagonists, particularly naftopidil, may induce neural suppression, in part by mediating hyperpolarization. The observed response was associated with glycinergic and/or GABAergic neural transmission, but may not depend on a1-adrenoceptors themselves. The present findings suggest that naftopidil may alleviate storage symptoms while also providing hyperpolarization in SG neurons, suppressing the micturition reflex by acting at the level of the spinal cord.

This study has some limitations. Although afferent nerves from the urothelium largely project to lamina $\mathrm{X}$ in the dorsal horn, SG neurons in lamina II were used. As described previously, a portion of the superficial neurons receives both nociceptive and nonnociceptive inputs from the lower urinary tract, as evidenced by upregulation of c-Fos expression [25]. Although the use of lamina $\mathrm{X}$ would be ideal, that structure has a low density of neurons, making the blind attachment of patchclamp electrodes more difficult. Instead, lamina II was exam- ined.

\section{AUTHOR CONTRIBUTION STATEMENT}

- Conceptualization: $D U$

- Formal Analysis: $D U, T H$

- Investigation: $D U, M Y$

- Methodology: $D U$

- Project Administration: $T H$

-Writing-Original Draft: $D U, T H$

-Writing-Review \& Editing: $D U, T H$

\section{REFERENCES}

1. Management of non-neurogenic male LUTS [Internet]. Arnhem (The Netherlands): European Association of Urology; 2018 [cited 2018 Dec 8]. Available from: https://uroweb.org/guideline/treatment-of-non-neurogenic-male-luts/.

2. Wei JT, Calhoun E, Jacobsen SJ. Urologic diseases in America project: benign prostatic hyperplasia. J Urol 2005;173:1256-61.

3. Takei R, Ikegaki I, Shibata K, Tsujimoto G, Asano T. Naftopidil, a novel alpha1-adrenoceptor antagonist, displays selective inhibition of canine prostatic pressure and high affinity binding to cloned human alpha1-adrenoceptors. Jpn J Pharmacol 1999;79:447-54.

4. Kwon SY, Lee KS, Yoo TK, Chung JI, Lee JY, Hong JH, et al. Comparison of the effect of naftopidil $75 \mathrm{mg}$ and tamsulosin $0.2 \mathrm{mg}$ on the bladder storage symptom with benign prostatic hyperplasia: prospective, multi-institutional study. Urology 2018;111:145-50.

5. Chen Z, Ishizuka O, Imamura T, Aizawa N, Igawa Y, Nishizawa O, et al. Role of alpha1-adrenergic receptors in detrusor overactivity induced by cold stress in conscious rats. Neurourol Urodyn 2009;28:251-6.

6. Gotoh M, Kamihira O, Kinukawa T, Ono Y, Ohshima S, Origasa H, et al. Comparison of tamsulosin and naftopidil for efficacy and safety in the treatment of benign prostatic hyperplasia: a randomized controlled trial. BJU Int 2005;96:581-6.

7. Nishino Y, Masue T, Miwa K, Takahashi Y, Ishihara S, Deguchi T. Comparison of two alpha1-adrenoceptor antagonists, naftopidil and tamsulosin hydrochloride, in the treatment of lower urinary tract symptoms with benign prostatic hyperplasia: a randomized crossover study. BJU Int 2006;97:747-51, discussion 751.

8. de Groat WC. Integrative control of the lower urinary tract: preclinical perspective. Br J Pharmacol 2006; 147 Suppl 2(Suppl 2):S25-40. 9. de Groat WC, Yoshimura N. Mechanisms underlying the recovery of lower urinary tract function following spinal cord injury. Prog Brain Res 2006;152:59-84. 
10. Takahara Y, Maeda M, Nakatani T, Kiyama H. Transient suppression of the vesicular acetylcholine transporter in urinary bladder pathways following spinal cord injury. Brain Res 2007;1137:20-8.

11. Sugaya K, Nishijima S, Miyazato M, Ashitomi K, Hatano T, Ogawa Y. Effects of intrathecal injection of tamsulosin and naftopidil, alpha-1A and -1D adrenergic receptor antagonists, on bladder activity in rats. Neurosci Lett 2002;328:74-6.

12. Sugaya K, Nishijima S, Kadekawa K, Ashitomi K, Ueda T, Yamamoto H. Spinal mechanism of micturition reflex inhibition by naftopidil in rats. Life Sci 2014;116:106-11.

13. Rexed B. The cytoarchitectonic organization of the spinal cord in the cat. J Comp Neurol 1952;96:414-95.

14. Yang K, Feng Y, Li Y. Baclofen inhibition of dorsal root-evoked inhibitory postsynaptic currents in substantia gelatinosa neurons of rat spinal cord slice. Brain Res 2001;900:320-3.

15. Yang K, Kumamoto E, Furue H, Li YQ, Yoshimura M. Action of capsaicin on dorsal root-evoked synaptic transmission to substantia gelatinosa neurons in adult rat spinal cord slices. Brain Res 1999; 830:268-73.

16. Jang IS, Rhee JS, Kubota H, Akaike N, Akaike N. Developmental changes in P2X purinoceptors on glycinergic presynaptic nerve terminals projecting to rat substantia gelatinosa neurones. J Physiol 2001;536(Pt 2):505-19.

17. Uta D, Xie DJ, Hattori T, Kasahara KI, Yoshimura M. Effects of naftopidil on inhibitory transmission in substantia gelatinosa neurons of the rat spinal dorsal horn in vitro. J Neurol Sci 2017;380:205-11.

18. Uta D, Hattori T, Yoshimura M. Effects of high concentrations of naftopidil on dorsal root-evoked excitatory synaptic transmissions in substantia gelatinosa neurons in vitro. Int Neurourol J 2018;22:252-9.
19. Yoshimura M, Jessell TM. Primary afferent-evoked synaptic responses and slow potential generation in rat substantia gelatinosa neurons in vitro. J Neurophysiol 1989;62:96-108.

20. Uta D, Furue H, Pickering AE, Rashid MH, Mizuguchi-Takase $H$, Katafuchi T, et al. TRPA1-expressing primary afferents synapse with a morphologically identified subclass of substantia gelatinosa neurons in the adult rat spinal cord. Eur J Neurosci 2010;31:1960-73.

21. Yoshimura M, Nishi S. Blind patch-clamp recordings from substantia gelatinosa neurons in adult rat spinal cord slices: pharmacological properties of synaptic currents. Neuroscience 1993;53:519-26.

22. Uta D, Hattori T, Yoshimura M. Characterization on responsiveness of excitatory synaptic transmissions to al-adrenoceptor blockers in substantia gelatinosa neurons isolated from lumbosacral level in rat spinal cords. Int Neurourol J 2019;23:13-21.

23. Mitsui T, Kanno Y, Kitta T, Moriya K, Nonomura K. Supraspinal Projection of serotonergic and noradrenergic pathways modulates nociceptive transmission in the lower urinary tract of rats. Low Urin Tract Symptoms 2016;8:186-90.

24. Kitta T, Mitsui T, Kanno Y, Chiba H, Moriya K, Yoshioka M, et al. Differences in neurotransmitter systems of ventrolateral periaqueductal gray between the micturition reflex and nociceptive regulation: An in vivo microdialysis study. Int J Urol 2016;23:593-8.

25. Birder LA, de Groat WC. Increased c-fos expression in spinal neurons after irritation of the lower urinary tract in the rat. J Neurosci 1992;12:4878-89.

26. Maeda A, Katafuchi T, Oba Y, Shiokawa H, Yoshimura M. Enhancement of GABAergic tonic currents by midazolam and noradrenaline in rat substantia gelatinosa neurons in vitro. Anesthesiology 2010;113:429-37. 